\title{
Lyme disease in the UK: epidemiology, clinical presentations and diagnosis
}

Lyme disease, a multisystem infection caused by a tick-borne spirochaete Borrelia burgdorferi, has intermittently received media attention in Britain during the past few years. What is known of its epidemiology and range of clinical presentations in the UK? Because the infection is not compulsorily notifiable in England and Wales, our information is based largely on voluntary reporting of patients with positive serological tests and experience gained in family practices and hospitals serving known endemic areas such as the New Forest. About 300 cases have been reported to the Public Health Laboratory Service (PHLS) Communicable Disease Surveillance Centre since 1986, but this is an underestimate of the true incidence, as some cases are not recognised, many settle spontaneously, having caused minor illness only, and some early cases are diagnosed and treated promptly without laboratory confirmation, especially in endemic areas.

Lyme disease has been acquired in many parts of the UK, including southern England from Kent to Devon, East Anglia, Yorkshire, Cumbria, the Welsh border country and the Scottish highlands. It is likely that ticks infected with $B$. burgdorferi are present in many areas with suitable ecological niches, including urban parklands. ${ }^{1}$ Ixodid (hard-bodied) ticks, commonly called sheep, dog or deer ticks, have a three-stage life cycle, feeding once during each stage, usually in the spring or autumn. Man can act as an incidental host for any stage. Larval and nymphal ticks are tiny and may not be noticed on the skin; therefore, a patient may not give a specific history of tick bite. Exposure to ticks may have been very brief, especially in citydwelling patients, e.g., during a picnic, day trip or holiday in the UK or abroad. Over $10 \%$ of cases serologically confirmed at the Lyme disease reference facilities of Southampton and Hereford Public Health Laboratories were contracted abroad, mainly in northern Europe and the USA. ${ }^{2}$

Clinical manifestations have been divided into three stages - early localised, early disseminated and late disease $;^{3}$ progression to late stages is not inevitable, even if treatment is not given. Patients with later manifestations may not recall having had a tick bite or rash, but a history of prior exposure to ticks should be carefully sought. Erythema migrans, spreading outwards from the site of a tick bite, is the most common early manifestation, occurring $2-30$ days after a tick bite, and may be accompanied by fever, headache and regional lymphadenopathy., ${ }^{4,5}$ These non-specific symptoms can occur without a rash.

During early disseminated infection, the organism spreads to other organs via the bloodstream or lymphatics, causing more severe systemic "flu-like" symptoms, arthralgia and sometimes multiple areas of erythema migrans. ${ }^{5}$ The most frequently reported manifestations of this stage are neurological, including lymphocytic meningitis, cranial nerve palsies (usually unilateral or bilateral facial palsy) and peripheral neuritis, occurring several weeks to several months after infection. Facial palsy is the second most commonly recognised clinical presentation of Lyme disease in children in England and Wales, erythema migrans being the most frequent. Radiculopathy, causing pain, sensory loss or weakness in the limbs or trunk, tends to present rather later, up to a year or more after initial infection. It can be debilitating because of chronic pain and cause a degree of disability if there is marked sensory loss. Nerve conduction defects may be demonstrated and conduction studies show a slow reponse to antibiotic treatment.

Neurological presentations of chronic Lyme disease with brain parenchymal involvement appear to be very rare in the UK. Clinical findings are not specific and may mimic the presenting features of multiple sclerosis, encephalitis, tumour, dementia or psychiatric illness. There is usually a pleiocytosis and detectable specific antibody in the CSF. Many of these patients have had earlier manifestations of Lyme disease. ${ }^{6}$

Although arthralgia is fairly common in the early disseminated stage, frank arthritis is unusual in cases acquired in the UK, in contrast to the USA where it is a frequent late manifestation, usually affecting large joints. There appears to be a significantly increased frequency of HLA DR4 haplotype amongst affected patients. ${ }^{5}$ Arthritis usually resolves after several years, but synovectomy is sometimes indicated.

Carditis, another manifestation of the early disseminated stage, is also more frequently seen in the USA, where cardiac complications, usually conduction defects, appear to occur in 4-10\% American cases. ${ }^{7}$ Cardiomyopathy is an uncommon but serious complication. Very few cases of cardiac complications have been reported in British patients, and several of those which have occurred were acquired abroad.

Acrodermatitis chronica atrophicans (ACA) is an uncommon late skin manifestation, reported more frequently from Europe than North America. Viable spirochaetes have been obtained from skin biopsies of ACA in which infection had occurred 12 years previously. ${ }^{4}$ The condition is usually seen on an extremity, with doughy textured blotchy discolouration and, eventually, shiny atrophic skin. There may be associ- 
ated peripheral neuropathy. The degree of response to antibiotic treatment depends on the extent of longstanding structural damage. Very few cases of ACA have been reported in the UK, but it is not known whether this is because the condition is rare or is not clinically recognised, as it may be mistaken for peripheral vascular insufficiency. ${ }^{4}$ Variations between American and European strains of $B$. burgdorferi may contribute to differences in patterns of disease presentation, but observer variation could also be a factor. ${ }^{4,8,9}$

Routine laboratory diagnosis is largely limited to antibody detection. Culture from biopsy material has a low success rate and may take longer than 6 weeks. Borrelial DNA detection by use of the polymerase chain reaction is likely to be more useful, especially in confirming suspected cases of erythema migrans, but it is not routinely available. ${ }^{10}$ Current antibody detection techniques include enzyme-linked immunosorbent assays with antigens derived from sonicated whole borreliae, flagellin-enriched preparations or purified flagellin. None will detect antibody within a few days of infection and, in some cases, antibody tests may remain negative for 6 weeks or longer, but they are rarely negative in disseminated or chronic Lyme disease. ${ }^{11}$

Prompt antibiotic treatment may ablate the antibody response, as experience with patients in the New Forest area has shown. During 1992, 11 cases of erythema migrans were confirmed serologically from this population. A further 17 cases were diagnosed clinically by a dermatologist or experienced general practitioner and treated within 3-7 days of onset but no serological response was detected by ELISA. Antibody tests based on recombinant antigenic flagellar epitope appear to be more sensitive and more specific in early infection but are not yet available commercially in the UK. ${ }^{12}$

Currently available ELISA tests may show crossreactions with antibody produced in response to other organisms, including oral spirochaetes and Treponema

\section{References}

1. Guy EC, Farquahar RG. Borrelia burgdorferi in urban parks. Lancet 1991 ; $338: 253$.

2. O'Connell S, Ferguson I, Guy E. Seroepidemiology of Lyme disease: UK experience. (Abstract) Fifth International Conference on Lyme Borreliosis, 1992. Arlington, VA, USA.

3. Åsbrink E, Hovmark A. Comments on the course and classification of Lyme borreliosis. Scand J Infect Dis 1991; 77 Suppl: $41-43$.

4. Åsbrink E. Cutaneous manifestations of Lyme borreliosis. Scand J Infect Dis 1991; 77 Suppl: 44-50.

5. Rahn DW. Lyme disease: Clinical manifestations, diagnosis, and treatment. Semin Arthritis Rheum 1991; 20: 201-218.

6. Pachner AR. Neurologic manifestations of Lyme disease, the new "great imitator". Rev Infect Dis 1989; 11 Suppl 6: S1482-S1486.

7. Cox J, Krajden M. Cariovascular manifestations of Lyme disease. Am Heart J 1991; 122 : 1449-1455.

8. Halperin JJ. North American Lyme neuroborreliosis. Scand $J$ Infect Dis 1991; 77 Suppl: 74-80. pallidum and to heat-shock proteins which are widely conserved in many species. Non-specific reactions may also be found in serum from patients with infectious mononucleosis, rheumatoid arthritis and other conditions. ${ }^{5,11}$ The use of immunoblot (Western blot) to assess the immune response to a range of $B$. burgdorfer $i$ antigens is helpful in confirming the specificity of a reactive ELISA test, but it may be necessary to look at sequential specimens in early illness to assess the evolving antibody response. Immunoblot is also useful in the investigation of CSF from patients with suspected neuroborreliosis, but the technique is labour intensive, difficult to standardise, and requires experience in performance and interpretation. Antibody test results cannot be interpreted fully without knowledge of the patient's clinical presentation, length of illness, antibiotic treatments and exposure risks. A positive result may indicate past infection rather than current disease, but this is likely to be a problem mainly for clinicians in endemic areas. ${ }^{11,13}$

Lyme disease is uncommon in the UK but may be acquired in many parts of the country where there are suitable habitats for ticks. Increased awareness of the condition in endemic areas is facilitating prompt treatment, which may lead to fewer patients exhibiting the later, more severe forms of the illness. However, the diagnosis may be missed, especially in patients who have no immediately obvious risk factors, because the clinical features can mimic many other conditions. The risk of acquiring Lyme disease can be minimised by applying commonsense measures to avoid tick bites, such as covering the limbs and using insect repellents when ticks are likely to be active and questing for blood meals. Ticks which have become attached should be removed promptly, as spirochaetal transmission from infected ticks is unlikely to occur within the first $24 \mathrm{~h}$ after attachment.

SUSAN O'CONNELL Public Health Laboratory, Southampton General Hospital, Southampton SO9 4XY.

9. Rosa PA, Hogan D, Margolis N. Molecular analysis of the major outer surface protein locus from a divergent Borrelia burgdorferi isolate from Europe. In: Schutzer SE (ed) Lyme disease: molecular and immunologic approaches. Cold Spring Harbor, NY, Cold Spring Harbor Press. 1992.

10. Schwartz I, Wormser GP, Schwartz JJ et al. Diagnosis of early Lyme disease by polymerase chain reaction amplification and culture of skin biopsies from erythema migrans lesions. J Clin Microbiol 1992; 30: 3082-3088.

11. Guy EC. The laboratory diagnosis of Lyme borreliosis. Rev Clin Microbiol 1993; 4: 89-96.

12. Magnarelli LA, Fikrig E, Berland R, Anderson JF, Flavell RA. Comparison of whole-cell antibodies and an antigenic epitope of Borrelia burgdorferi in serologic tests for diagnosis of Lyme borreliosis. J Clin Microbiol $1992 ; 30$ : 3158-3162.

13. Guy EC, Bateman DE, Martyn CN, Heckels JE, Lawton NF. Lyme disease: prevalence and clinical importance of Borrelia burgdorferi specific IgG in forestry workers. Lancet $1989 ; 1$ : 484-486. 